\title{
MÖSSBAUER SPECTROSCOPY SYSTEM WITH INCREASED PERFORMANCE AND FLEXIBILITY UTILIZATION IN MATERIAL RESEARCH
}

\author{
Jiří Pechoušek ${ }^{*}$ - Petr Novák ${ }^{*}$ - Jakub Navařík ${ }^{*}$ \\ Pavel Kohout ${ }^{* *}$ _ Libor Machala ${ }^{*}$
}

\begin{abstract}
A new concept of Mssbauer spectrometer used for material research is presented. Performance of this computer based measurement system was carefully analyzed and programming code of the application step by step optimized. By implementation of parallel data processing the control application with zero dead-time of measurement was developed.

K e y w or ds: Mössbauer spectrum, Mössbauer spectrometer
\end{abstract}

\section{INTRODUCTION}

Measurement efficiency of the modern spectroscopy systems is determined by the quality of the hardware used to acquire signals and by the software used to analyse data. Up-to-date systems process data with zero data amount loss. In this paper, a new concept of Mössbauer spectrometer used for material research is presented.

The Mössbauer effect is based on recoilless nuclear emission and resonant absorption of gamma-rays in the sample. The Mössbauer spectra acquisition is performed by gamma-ray intensity measurement together with radioactive source motion control causing the Doppler shift of the gamma-ray photons $[1,2]$. Mössbauer spectrometer consists of spectrometric bench (radioactive source, velocity transducer, sample holder, and gamma-ray detector), measurement hardware, and control application. The spectrometer has to perform tasks such as gammaray pulse-height analysis, reference velocity signal generation for the motion of the source, proportional integral derivative (PID) control of the relative velocity between the source and the absorber, and Mössbauer spectra accumulation. Mainly the synchronization between the detector signal acquisition and the source velocity motion processes is of crucial importance in this system. High precision execution of the detector signal analysis and the source movement control tasks is reflected in the quality of the system.

Employing Virtual Instrumentation (VI) concept (implemented in National Instruments, NI, LabVIEW ${ }^{\text {тм }}$ programming environment) for developing measurement instruments, a unique Mössbauer spectrometer has been built [3-8]. Besides the commercial products, we use other own developed units (gamma-ray detectors, signal amplifiers, velocity transducers ... ). For many years, we ap- ply the VI concept in the design of our spectrometers, and with new functions, techniques, and processes implemented in LabVIEW, the system is improved continually.

The improved Mössbauer spectrometer measurement system offers a new approach to Mössbauer spectrometer construction. By detailed analyses and optimization we designed a high-performance system, which is generally able to save the measurement time. VI solution significantly increases the system performance and flexibility reflected in easy customisation for using cryostats, furnaces and other equipment necessary for a complex sample analysis.

The process of the dead time decreasing of the fullyLabVIEW based Mössbauer spectrometer [4] is presented by the use of advanced fetching options in software driver, FIFO (first-in-first-out) memory programming techniques, and trigger occurring analysis. A selected utilization of the spectrometer is presented in the last section.

\section{MÖSSBAUER SPECTROSCOPY}

Transmission Mössbauer Spectroscopy (TMS) is used for structural, phase, and magnetic characterization of mostly iron containing samples (commonly Fe, Sn, Ni, $\mathrm{Zn}, \mathrm{Eu}$ etc.). Mössbauer Spectroscopy (MS) is a highly element selective method and in details it provides determination and quantification of phase composition of samples including amorphous and nanocrystalline; determination of valence and spin states of iron atoms, differentiation of structure positions of iron atoms, stoichiometry examination of cation substitution; magnetic state determination and local configuration of magnetic moments of the atoms; measurement of temperature dependences,

\footnotetext{
* Regional Centre of Advanced Technologies and Materials (RCPTM), Department of Experimental Physics, Faculty of Science, Palacky University, 17. listopadu 12, 77146 Olomouc, Czech Republic; jiri.pechousek@upol.cz

** Department of Experimental Physics, Faculty of Science, Palacky University, 17. listopadu 12, 771 46, Olomouc, Czech Republic
} 


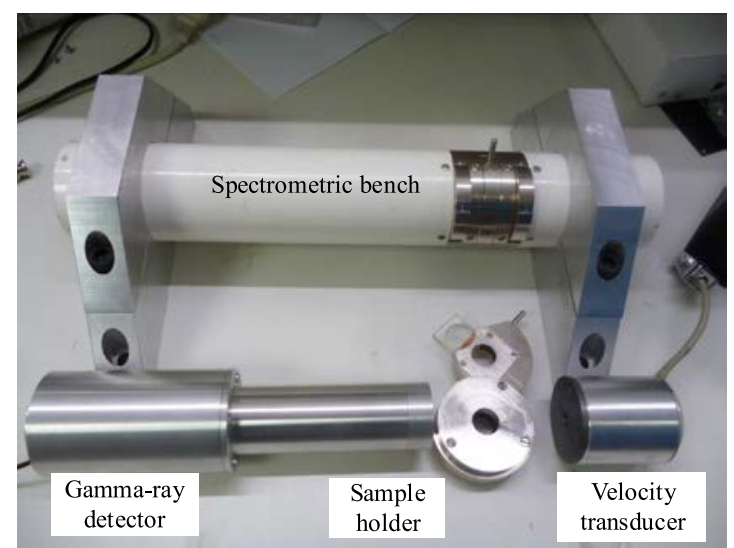

Fig. 1. Mössbauer spectrometric bench for room temperature measurements

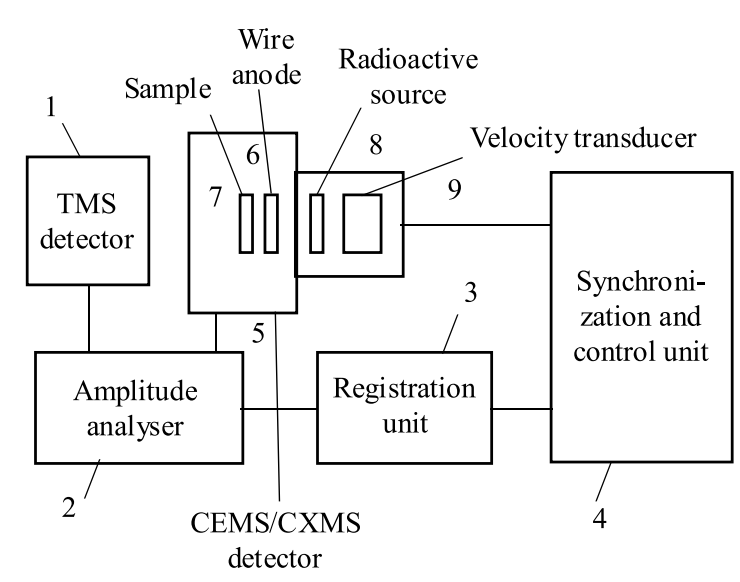

Fig. 2. Mössbauer spectrometric bench for room temperature CEMS/CXMS/TMS measurements

measurement in external magnetic field; determination of magnetic properties including temperatures of magnetic transitions; and study of mechanism and kinetics of reactions in solid phase, phase transformations. The spectrometric bench for room temperature TMS measurements is presented in Figure 1.

This compact bench allows performing a precise analysis of the samples in TMS mode. The main parts include a gamma-ray detector, sample holder with collimators and velocity transducer.

Conversion Electron Mössbauer Spectroscopy (CEMS) and Conversion X-ray Mössbauer Spectroscopy (CXMS) are a pair of methods, which help investigate Mössbauer radiation in the backscattering geometry. Considering the energy losses during non-elastic collisions in the examined material is the scope of conversion electrons, or conversion X-ray radiation (originating from the sample surface of $\sim 300 \mathrm{~nm}$, resp. $1-10 \mu \mathrm{m}$ thickness). Therefore, this technique is suitable for studying thin films and surface phase composition of the materials. The block diagram of the spectrometric bench for room temperature CEMS/CXMS/TMS measurements is in Fig. 2.
All methods act as supporting methods in physics of materials, nanotechnology, metallurgy, chemistry, archaeometry, geology, and mineralogy.

If cryostats and furnaces are included, it is possible to analyse samples at different conditions, such as low temperatures (ie from 1.5 up to $300 \mathrm{~K}$ ) and high temperatures ( $i e$ up to $1000^{\circ} \mathrm{C}$, with a possibility of inert atmosphere) and under an external magnetic field of up to $8 \mathrm{~T}$ (in the low temperature mode) [8]. The samples can be in the form of powder as well as thin films (plates). In the case of bigger compact samples, a backscattering mode of Mössbauer spectroscopy can be applied for characterization of the surface of the sample.

\section{SPECTROMETER AS AN INSTRUMENT WITH ZERO MEASUREMENT DEAD TIME}

Mössbauer spectrometer as a virtual instrument [48] consists of spectrometric bench, measurement hardware (Windows-based computer, high-speed digitizer, and function generator), and application fully developed in LabVIEW ${ }^{\text {тм }}$.

There are two main devices in this computer-based spectrometer; the first one is the digitizer (NI PCI-5124), and the second, the function generator (NI PCI-5401). The function generator works as a master, and except the periodic analog signal (reference velocity) on its signal output, it generates the digital trigger signal with the same frequency on the synchronization output. This signal is programmatically routed via internal Real Time System Integration (RTSI) bus line to control high-speed digitizer which works as a slave. The gamma-ray detection and analysis system uses high-speed digitizer. The spectra accumulation process combines the radioactive source velocity data with the corresponding gamma-ray intensity transmitted thorough the sample (in the transmission mode of measurement). The velocity signal frequency is mostly up to tens of hertz $(20-50 \mathrm{~Hz})$.

Detector signal is digitized by the digital oscilloscope with given sampling rate. Its value corresponds with pulse length, activity of the source etc.; however, generally the value of $10 \mathrm{MS} \mathrm{s}^{-1}$ (mega samples per seconds) is suitable for common utilization [8]. With sampling rate value and frequency of the velocity signal correspond also the number of samples per one period of the velocity signal. Those samples as one data block are then processed by algorithms for pulse processing, amplitude analysis and discrimination. Mentioned algorithms have to be as much as fast possible to process data during one period of the velocity signal. For example, in the case of velocity signal frequency of $30 \mathrm{~Hz}$, sampling rate of $10 \mathrm{MS} \mathrm{s}^{-1}$ for detector signal acquisition, one period takes approx. $33 \mathrm{~ms}$ and 333333 samples.

The spectra accumulation process is based on the periodical summation of the appropriate data from each repetitive movement period for hours or days. Each period of the source movement is divided into 2,048 velocity/time intervals. The number of the detected photons 


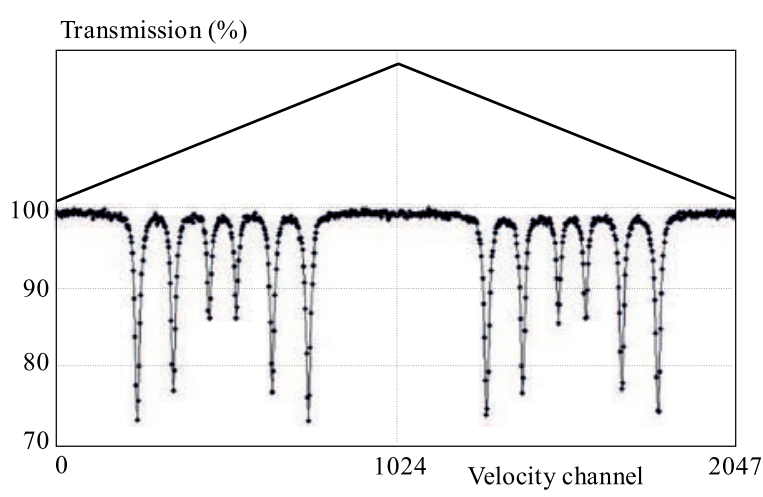

Fig. 3. Spectra accumulation process during many periods of the reference velocity signal (depicted at the top)

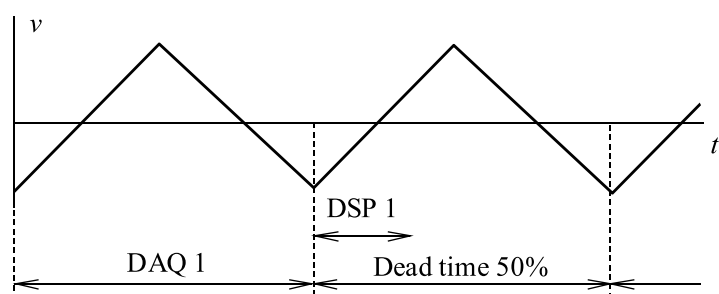

(a)

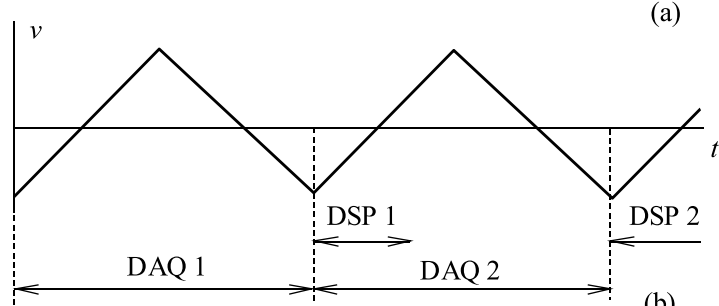

(b)

Fig. 4. Serial a - behaviour of the DAQ and DSP tasks and b implementation of the parallelism for for one period of the velocity signal

accumulated during each time interval is saved into the relevant velocity channel, see typical calibration spectrum in Fig 3.

In the main LabVIEW code the generator software instrument driver is used in easy setup way; after the initialization and configuration processes, the signal generation is started. At the end of the generation, the abort function is called and the instrument session is closed.

First version of the digitizer-based code was built as simple as possible in a high-level setup. Unfortunately, with this code the highest dead time value of the system was achieved (more than 50 percent). The block diagram is based on the basic programming flow for using digitizer functions, initialization, configuration, reading the data, and abort. Crucial triggering function is implemented in the code. The trigger transfers a device from a nonsampling into a sampling state. After initializing an acquisition, the digitizer waits for the start trigger, and the device starts acquiring data upon receiving the start trigger. The pulse coming from a detector is acquired with the internal function, which retrieves data that the digitizer has acquired and returns a one-dimensional array of binary 8-bit values.
After the performance analysis of this code, an advanced code optimized for fetching as fast as possible was used; hence the device is basically setup to acquire data forever. This fetching is complex and application is high throughput. The application reads the available record and stores in the digitizer onboard memory before fetching data. As a further improvement a FIFO was implemented, and the fastest way with pushing data into a software queue in one programmatic loop named DAQ (data acquisition) and dequeueing it in second loop named DSP (digital signal processing) was utilized. Both loops implement parallel behaviour. DSP loop waits for data from DAQ loop, which transfers data into the FIFO memory. In the case of slow behaviour of the DSP loop (DSP algorithm is not able to process data in time), FIFO is filled and data are processed later (after few periods) when computer processor has lower usage.

A value of approx. 20 percent of the dead time was reached, however, no all records the code is able to read from the onboard memory even the DSP algorithm takes approx. $10 \mathrm{~ms}$ to process data (see above mentioned example).

After the trigger occurrence analysis, the length of the records was slightly reduced of 5 percent at the end of data block. The deleted portion of data is not used generally for spectra accumulation. With this concept, all triggered events were registered, and no detector signal was lost, hence zero dead time was reached.

It is necessary to mention that zero dead time was reached in the PCI version of devices, for clarity. In the case of USB version of device where 50 percent of dead time was reached, low-level programming method is not able to implement since those functions are not supported in the instrument drivers for this type of devices.

\section{AN EXPERIMENTAL STUDY WITH THE CEMS MODE}

As a representative example of power of ${ }^{57} \mathrm{Fe}$ Conversion Electron Mössbauer Spectroscopy, we present a task to determine amount of austenite (gamma-Fe(C) phase) in the sample of stainless steel. Austenite is used to be formed during a manufacture of stainless steel and its higher content can affect mechanical properties of the sample. The sample was too thick to analyse it by conventional TMS, therefore CEMS was used. With respect to expected homogenous phase composition within the whole volume of the sample, CEMS measurement could provide a characterization of all the sample, despite the data were collected only from a thin surface layer (less than $300 \mathrm{~nm}$ ). The CEMS spectrum, represented by experimental points, overall fits, and individual fitted components, is depicted in Fig. 5. Table 1 provides a summary of the values of the Mössbauer parameters and assignments of individual components.

In Tab. $1 \delta$ is the isomer shift, $\Delta E_{q}$ is the quadrupole splitting, $B_{\mathrm{hf}}$ is the hyperfine magnetic field, $\Gamma$ is the 


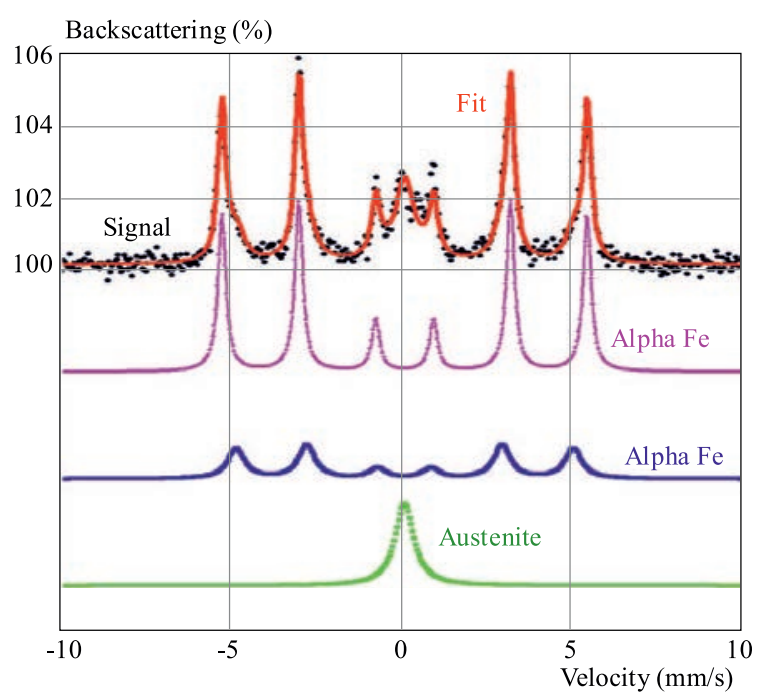

Fig. 5. Mössbauer (CEMS) spectrum of stainless steel sample

Table 1. Mössbauer parameters and relative contents of components identified in the CEMS spectrum

\begin{tabular}{|c|c|c|c|c|c|c|}
\hline \multirow[b]{2}{*}{ Phase } & \multicolumn{6}{|c|}{ Mössbauer parameter } \\
\hline & Component & $\begin{array}{c}A(\%) \\
\pm 0.5\end{array}$ & $\begin{array}{c}\delta \\
(\mathrm{mm} / \mathrm{s}) \\
\pm 0.01\end{array}$ & $\begin{array}{c}\Delta E_{q} \\
(\mathrm{~mm} / \mathrm{s}) \\
\pm 0.01\end{array}$ & $\begin{array}{c}B_{\mathrm{hf}} \\
(\mathrm{T}) \\
\pm 0.3\end{array}$ & $\begin{array}{c}\Gamma \\
(\mathrm{mm} / \mathrm{s}) \\
\pm 0.01\end{array}$ \\
\hline alpha-Fe & sextet & 59.7 & 0.00 & 0.00 & 33.0 & 0.27 \\
\hline alpha-Fe & sextet & 24.9 & 0.01 & 0.01 & 30.5 & 0.57 \\
\hline austenite & singlet & 15.5 & 0.00 & - & - & 0.65 \\
\hline
\end{tabular}

linewidth, and $A$ is the area of the subspectrum. The results show that Mössbauer spectroscopy is able to discern and quantify two different alpha zero valent iron (alphaFe) based phases in the stainless steel. These two phases are represented by two sextets with almost the same values of isomer shift and quadrupole splitting but differing in the hyperfine magnetic field. In addition to the major (alpha-Fe) phases, there are about $15.5 \%$ of gammaFe, which is expected to be in a solid mixture with carbon forming the austenite phase. X-ray powder diffraction could also give a quantification of austenite phase, however, not so precisely like Mössbauer spectroscopy.

\section{DISCUSSION}

Main advantage of the presented concept is utilization of measurement hardware type. In this case, Windowsbased computer, high-speed digitizer, and function generator are used to realize signal acquisition. A control application performing data analysis and spectra accumulation with user friendly application was then developed.
The main application is hardware-platform independent, since it is usable with USB, PCI, PCIe, PXI, and PXIe modules used for signal acquisition, and is able to apply it in different Mössbauer techniques (TMS, CEMS, CXMS, other backscattering, etc).

The control application was optimized for reaching zero dead-time of measurement by implementation of parallel data processing and by using of fast pulse processing algorithms.

\section{Acknowledgement}

The authors gratefully acknowledge the support by the Operational Program Research and Development for Innovations - European Regional Development Fund (project CZ.1.05/2.1.00/03.0058 of the Ministry of Education, Youth and Sports of the Czech Republic) and the Operational Program Education for Competitiveness European Social Fund (projects CZ.1.07/2.3.00/20.0017, CZ.1.07/2.3.00/20.0155, and CZ.1.07/2.3.00/20.0058 of the Ministry of Education, Youth and Sports of the Czech Republic). Financial support from the internal student grant IGA of Palacky University in Olomouc, Czech Republic, (PrF_2013_014) is gratefully acknowledged.

\section{REFERENCES}

[1] MADDOCK, A. G.: Mössbauer Spectroscopy: Principles and Applications of the Techniques, Woodhead Publishing, Cambridge, United Kingdom, 1997.

[2] GREenWOOD, N. N.-GIBB, T. C. : Mössbauer Spectroscopy, Chapman and Hall, London, United Kingdom, 1971.

[3] MASHLAN, M.-JANCIK, D.-ZAK, D.-DUFKA, F.-SNASEL, V.-KHOLMETSKII, A. L. : In Mössbauer Spectroscopy in Materials Science 1998 (M. Miglierini and D. Petridis, eds.), Kluwer Academic Publishers, Dordrecht, Netherlands, 1999, p. 399.

[4] PECHOUSEK, J.-PROCHAZKA, R.-JANCIK, D.-MASHLAN, M.-FRYDRYCH, J.: Journal of Physics, Conference Series 217 (2010), 012006.

[5] PECHOUSEK, J.-JANCIK, D.-EVDOKIMOV, V.-PROCHAZKA, R.: Nuclear Instruments and Methods in Physics Research B 267 (2009), 846.

[6] FRYDRYCH, J.-MASHLAN, M.-ZBORIL, R.-PECHOUSEK, J.-HERMANEK, M.: Acta Metallurgica Slovaca 13 (2007), 298.

[7] PEChOUSEK, J.-PROCHAZKA, R.-PROCHAZKA, V.FRYDRYCH, J. : Nuclear Instruments and Methods in Physics Research A 637 (2011), 200.

[8] PECHOUŠEK, J.-JANČÍK, D.-FRYDRYCH, J.-NAVAŘÍK, J.-NOVÁK, P.: AIP Conference proceedings 1489 (2012), 186.

Received 14 April 2013

Jiří Pechoušek, Petr Novák, Jakub Navařík, Pavel Kohout, Libor Machala - biographies not supplied. 